\title{
Personalized vaccines use tumour fingerprint to target glioblastoma
}

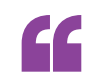

\section{Glioblastoma}

presents one

of the greatest

challenges

in modern

oncology

g

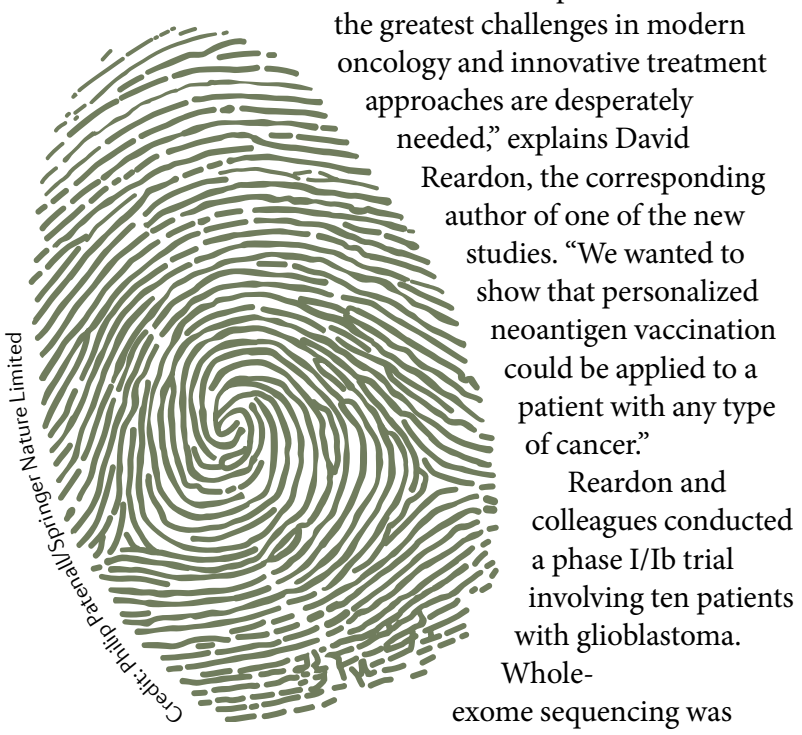

carried out to identify mutations in patient tumour cells, and gene expression was confirmed by RNA sequencing analysis. Personalized vaccines were then generated against a subset of mutant epitopes produced by each patient.

The neoantigen vaccine was safe and produced no serious adverse effects. Vaccination failed to elicit a response in patients who received the immunosuppressant agent dexamethasone, but yielded a strong antitumour immune response in two of the remaining patients. These two individuals generated neoantigen-specific $\mathrm{T}$ cells that were able to cross the blood-brain barrier and traffic to the tumour in the brain.

The results suggest that personalized neoantigen vaccination is a promising therapy for glioblastoma, but the authors note that further trials are needed to confirm the efficacy of this approach and to determine its place among other treatments. "The next step is to combine this therapy with other treatments that are designed to overcome some of the immunosuppressive strategies exploited by glioblastoma tumours that can prevent antitumour immune responses from impacting the tumour," concludes Reardon.

A second phase I study of personalized vaccination in glioblastoma was published in the same issue of Nature. The Glioma Actively Personalized Vaccine Consortium (GAPVAC), a multicentre initiative spanning six European clinical sites, enrolled 15 patients with glioblastoma and tested two types of vaccine.

The first vaccine type, APVAC1, was targeted against a previously constructed library of non-mutated antigens that are over-represented in glioblastoma tumours. The second type, APVAC2, was mainly targeted against mutated neoantigens. However, if no suitable neoantigens were identified in a patient, the vaccine was instead targeted against non-mutated antigens that were not present in the pre-made library. The composition of both vaccines was personalized according to the results of microarray analysis of the patient transcriptome and mass spectrometry analysis of their HLA immunopeptidome.

Both vaccine types showed favourable safety results and elicited $\mathrm{T}$ cell responses against the proteins in the vaccine. APVAC1 induced a sustained $\mathrm{CD}^{+} \mathrm{T}$ cell response, and APVAC2 induced both $\mathrm{CD} 4^{+}$and $\mathrm{CD}^{+} \mathrm{T}$ cell responses.

"This study demonstrates the feasibility of a highly personalized form of immunotherapy in a multicentre, multinational clinical setting," concludes Wolfgang Wick, the corresponding author of the study. Wick and colleagues also suggest that future work might examine the combination of personalized vaccinations with adjuvants and checkpoint inhibitors.

Charlotte Ridler

ORIGINAL ARTICLES Keskin, D. B. et al. Neoantigen vaccine generates intratumoral T cell responses in phase lb glioblastoma trial. Nature https://doi.org/10.1038/s41586-018-0792-9 (2018) | Hilf, N. et al. Actively personalized vaccination trial for newly diagnosed glioblastoma. Nature https://doi.org/10.1038/ s41586-018-0810-y (2018) FURTHER READING Weller, M. et al. Vaccine-based immunotherapeutic approaches to gliomas and beyond. Nat. Rev. Neurol. 13, 363-374 (2017) 